\title{
SIMPA: Soft-Grasp Infant Myoelectric Prosthetic Arm
}

\author{
D. De Barrie, R. Margetts, and K. Goher
}

\begin{abstract}
Myoelectric prosthetic arms have primarily focused on adults, despite evidence showing the benefits of early adoption. This work presents SIMPA, a low-cost 3D-printed prosthetic arm with soft grippers. The arm has been designed using CAD and 3D-scanning, and manufactured using predominantly 3D-printing techniques. A voluntary opening control system utilizing an armband-based sEMG has been developed concurrently. Grasp tests have resulted in an average effectiveness of $87 \%$, with objects in excess of $400 \mathrm{~g}$ being securely grasped. The results highlight the effectiveness of soft grippers as an end device in prosthetics, as well as the viability of toddler scale myoelectric devices.
\end{abstract}

Index Terms - Prosthetics and Exoskeletons, Soft Robot Applications, Additive Manufacturing.

\section{INTRODUCTION}

$\mathrm{U}$ PPER limb reduction defects occur congenitally in 4.1-5 per 10,000 births [1]. Factoring in non-congenital amputations is problematic, though one long term study showed dysvascular, trauma-related, and cancer-related conditions had a respective frequency of $2.25,2.65$, and 0.15 per 100,000 between the ages of 0-14 years [2]. Despite these figures, active prosthetic devices are routinely only given to adults, with the assumption that myoelectric devices (those controlled by electrical signals generated in the muscles) are difficult to scale down, as well as being too expensive, especially with the frequent replacement schedule that a growing child necessitates [3].

In cases where young children with upper limb amputation (ULA) utilize a prosthetic device, the child will often develop their own methods of grasping objects [4]. This causes later difficulty adapting to methods using a prosthetic device as the child's motor neural skills and proprioception will have only developed up to the base of the stump. This adaptive grasping can also cause physiological issues in the long term, such as asymmetric posture and muscular-skeletal pain [5] due to an overreliance on the residual limb and off balance center of mass [6]. Despite the benefits of prosthetic use, rejection

Manuscript received September, 10, 2019; Revised December, 7, 2019; Accepted December, 17, 2019.

This paper was recommended for publication by Editor P. Valdastri upon evaluation of the Associate Editor and Reviewers' comments.

Daniel De Barrie, Rebecca Margetts, and Khaled Goher are with the School of Engineering, University of Lincoln, United Kingdom. ddebarriedincoln.ac.uk

Digital Object Identifier (DOI): see top of this page. remains a major issue. Early fitting has been shown to reduce this risk [7], with one study showing a rejection rate for fitting before and after the age of 2 years of $22 \%$ and $58 \%$ respectively [8]. If the usefulness of the device is demonstrated to the child, the rejection rate is greatly reduced [9]; a functional myoelectric device should therefore aid in reducing rejection rates.

The cost of an active prosthetic in high-income nations, such as the USA, is upwards of $\$ 20,000$ [10], with even simple cosmetic options costing around $\$ 3,000-\$ 5,000$. In low-income nations an expense on this scale for a custom fit prosthetic device is totally unfeasible, especially with many families already facing hardship as a result of the amputation [11]. The use of additive manufacturing introduces the prospect of rapidly producing low-cost custom prosthetic devices, such as the Rehand [12] with a production cost below $\$ 1250$; this cost may be reduced further should 3D-printing be used in conjunction with injection molding for standardized parts [13]. The technology has already been proven as means of producing myoelectric prosthetics [14], though thus far the pediatric devices have predominantly been open-source bodypowered devices, with very limited functionality.

This work presents a myoelectric device for toddlers that can be produced at a low cost, whilst maintaining high grasp performance levels. To achieve this, cable-driven soft-grippers have been integrated into the design, with the intention of improving the grasp contact surface. The soft grippers also aim to provide a more even distribution of the grasp force, mimicking the grip force distribution of a human hand [15]. The device has been named SIMPA: Soft-grasp Infant Myoelectric Prosthetic Arm.

\section{Prosthetic Design AND REALIZATION}

The prosthetic device considered in this work was designed using Autodesk Inventor 2019 (Autodesk, Inc.), with the intention of using fused deposition modelling (FDM) 3Dprinting for the main structure, only sourcing additional components where necessary. The Ultimaker S5 (Ultimaker B.V.) was used to produce the parts due to its large print area and dual material extrusion. The dimensions of the arm are based on data, sourced from a volunteer, which represents the size of a 4-year-old male forearm. A 3D-scan of the stump used for modelling was also sourced from this individual. Socket modelling utilized the 'Mesh' feature along with a 3Dscan of the stump, to create an accurate socket that can then be 3D-printed (Figure 1). This avoids the need for traditional 
stump plaster-casting, a process that is both time consuming and often uncomfortable to the individual.
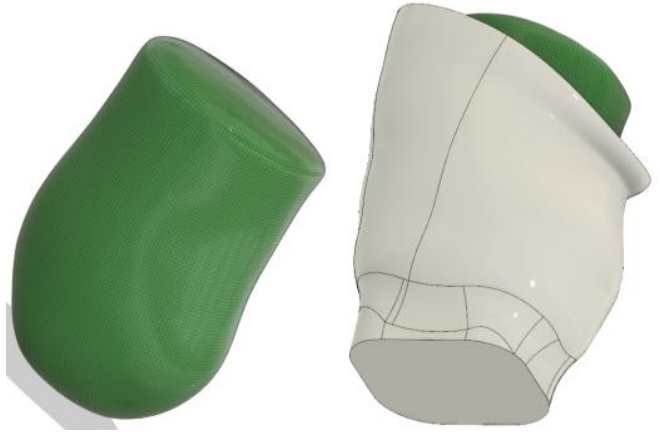

Fig. 1. 3D- Mesh (left), Constructed Socket CAD Model (right)

The end device of prosthetic utilizes wire-driven soft grippers, loosely based on the appearance of human fingers. The grippers are a composite, manufactured out of two different silicon rubbers. The malleable material Dragon Skin $^{\mathrm{TM}} 30$ (Smooth-On, Inc.) acts as the grip surface, whilst the more rigid Smooth-Sil ${ }^{\mathrm{TM}} 960$ (Smooth-On, Inc.) provides the elastic tension required to return the hand to its open position once tension is released from the cables.

The grippers were formed using a 3D-printed mold. This mold was printed in slightly flexible TPU-95, to ensure easy removal of the component. In the final device, three grippers are used: a two-segment 'thumb' and, two three-segment 'fingers' (Figure 2) offset $90^{\circ}$ from the 'thumb'. A variant with a three-segment 'thumb' was also produced. The decision to use three fingers, rather than five, was predominantly due the difficultly in manufacturing grippers small enough for five to fit within the $50 \mathrm{~mm}$ width of the hand. The use of three grippers in robotics has been shown to achieve a stable grasp rate of $90 \%$ [16] and this was deemed sufficient, given the size restrictions in place.

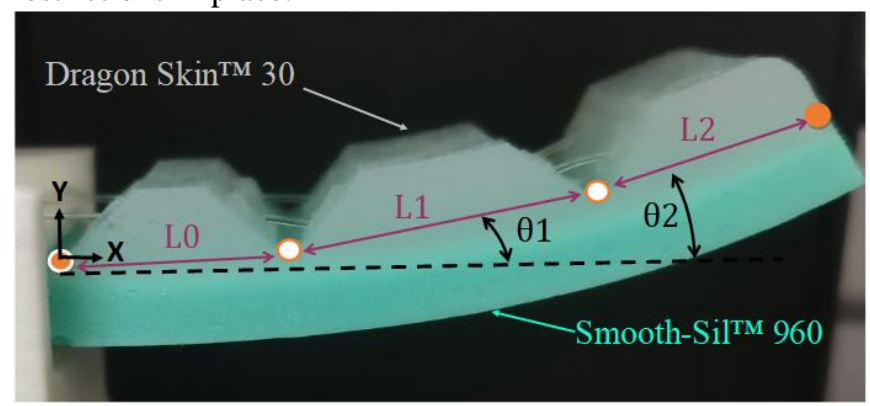

Fig. 2. 3-Segment Soft Gripper

The kinematics of the grippers is defined in Equations (1) and (2), using the variables displayed in Fig. 2.

$$
\begin{aligned}
& x=l_{0_{x}}+l_{1} \cos \left(\theta_{1}\right)+l_{2} \cos \left(\theta_{2}\right) \\
& y=l_{0_{y}}+l_{1} \sin \left(\theta_{1}\right)+l_{2} \sin \left(\theta_{2}\right)
\end{aligned}
$$

These equations are based on the three-segment gripper, for the two-segment variant $l_{2}$ and $\theta_{2}$ would be defined as 0 . As the grippers are by design flexible, there is a lateral deformation of up to approximately $\pm 15^{\circ}$ that is not defined in these kinematic equations. This deformation occurs only went contact with an object has occurred, as it relative to surface's geometry. Figure 3 shows the hand in a semi-closed and closed position to highlight the soft-grippers' range of motion.

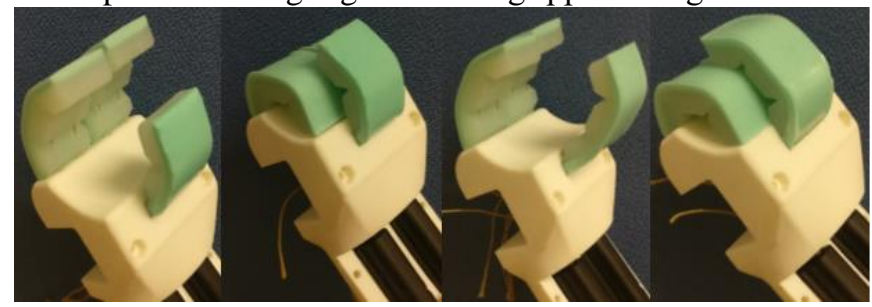

Fig. 3. From Left to Right: 2-Segment Thumb Semi-Closed, 2-Segment Thumb Closed, 3-Segment Thumb Semi-Closed, and 3-Segment Thumb Closed

The main body of the arm was printed in Acrylonitrile Butadiene Styrene (ABS), a strong and durable polymer, commonly used in functional additively manufactured products. The arm houses two Actuonix PQ12-P Micro Linear Actuators (Actuonix Motion Devices, Inc.), which provide a maximum driving force of $30 \mathrm{~N}$ each. One solely actuates the 'thumb', whilst the second drives the two 'fingers'. The actuators contain a built-in potentiometer for determining the extension of the shaft. The system controller is an Arduino Nano which, along with a motor driver, controls the two actuators. The system is powered by a $7.5 \mathrm{~V}$ Lithium-Ion battery placed close to the socket. The system draws $2.5 \mathrm{~W}$ and $0.5 \mathrm{~W}$ during actuation and rest respectively. This allows for between 6.6 and 33 hours of use with the rated $16.5 \mathrm{Wh}$ battery, depending on the number of actuations. The user operates the system via surface-electromyography (sEMG). Typically, sensor pads would be attached to the skin via an adhesive. The present design, however, utilizes the OYMotion Gravity: Analog EMG Sensor (OYMotion Technologies, Inc.). This armband-based device allows the user to quickly attach and detach the device, whilst providing accurate sEMG recordings.

The prototype device comprises three 3D-printed components assembled using M3 bolts (Figure 4). All of the electrical components are housed inside the body the arm, excluding the sEMG armband. The total weight of the device, including the armband, is around $395 \mathrm{~g}$. For comparison, the approximate weight of a biological forearm is around $314 \mathrm{~g}$ for a 4 -year-old male [17], [18].

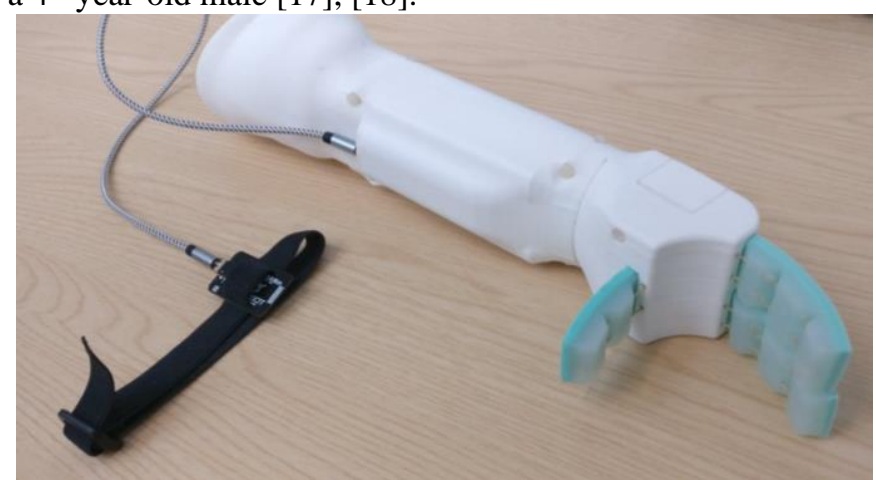

Fig.4. Functional SIMPA Prototype

A certain level of modularity is present in the device (Figure 5). All of the electrical components and grippers could be transferred to a new printed arm once the child has outgrown the current one. The soft-grippers too can easily be 
replaced, should they become damaged or excessively worn.

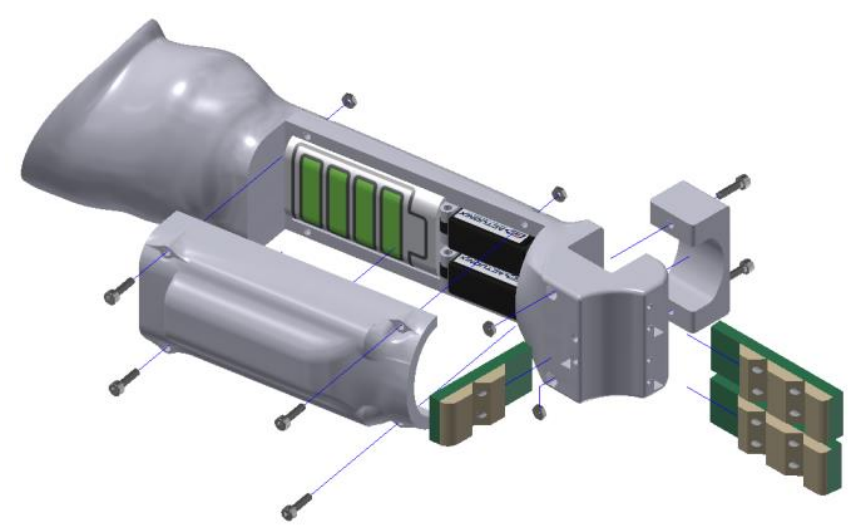

Fig. 5. Exploded CAD Model

\section{CONTROL SYSTEM DESIGN}

The control system utilizes an Arduino Nano based circuit (Figure 6). The system is controlled by the user via the armband-based sEMG unit. The actuators provide position feedback using built-in potentiometers. A motor driver and voltage regulator are also incorporated into the system.

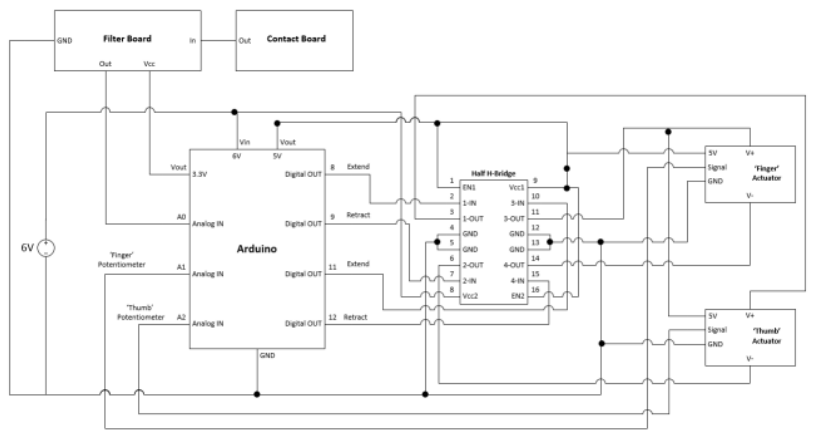

Fig. 6. Circuit Layout

A voluntary opening single site sEMG control system was utilized. This style of system is given the term 'cookiecrusher' in [19] and is the most simplistic form of myoelectric prosthetic control: it is intended to be simple as it would likely be a child's first device, acting as a building block for more advanced, multi-site, multi-action systems. This single-site approach was also used in [3], one of the only studies demonstrating prosthetic use in young children. The prosthetic could, with relative ease, be reconfigured for this multi-site approach should this perform better with the end user. The system was designed using Simulink Support Package for Arduino Hardware (The MathWorks, Inc.). The system can be broken down into two constituent parts, the sEMG and the grasp detection.

The OYMotion Gravity was used to record the sEMG signals. The armband was attached approximately at the center of the bicep. The sEMG location is due to this prototype design being based around a high-level transradial amputee, with an insufficiently sized residual limb for forearm-based recording. The author, a 23-year-old male of average build, was used during the development and initial testing of the system, in order to prove the concept. Future validation with an age-appropriate subject is planned. Raw data was collected of the muscle flexing and relaxing over a period of approximately 10s, as shown in Figure 7. The plots were all created in real time using scopes at significant locations on the Simulink model. The serial refresh rate was set to $0.01 \mathrm{~s}$, as this provides enough accuracy without being too computationally demanding.

The raw recording averages around 300 on the Arduino's analog input scale (10-bit ADC, 0-1023). The raw data was first normalized around 0 and set to an absolute scale, so that the activity is contained within the positive region. This normalized data still contains a large amount of noise. A moving average filter has been incorporated here to smoothen the data, resulting in the plot shown in Figure 8.

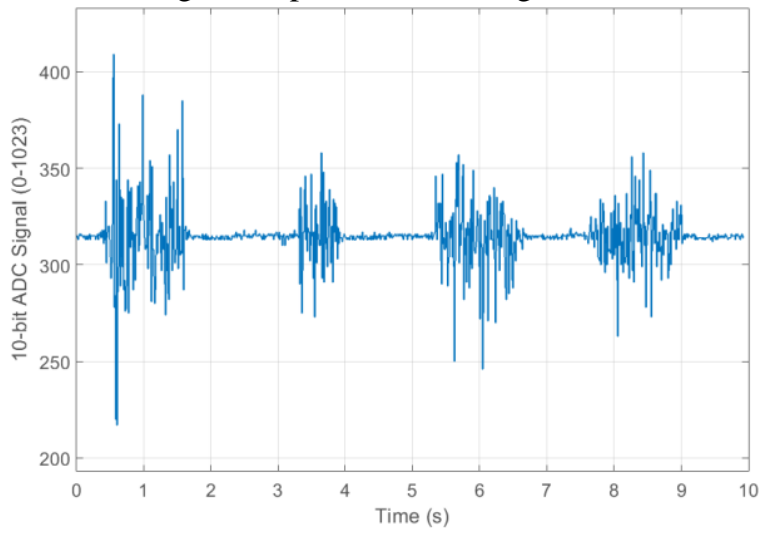

Fig. 7. Raw sEMG Recording

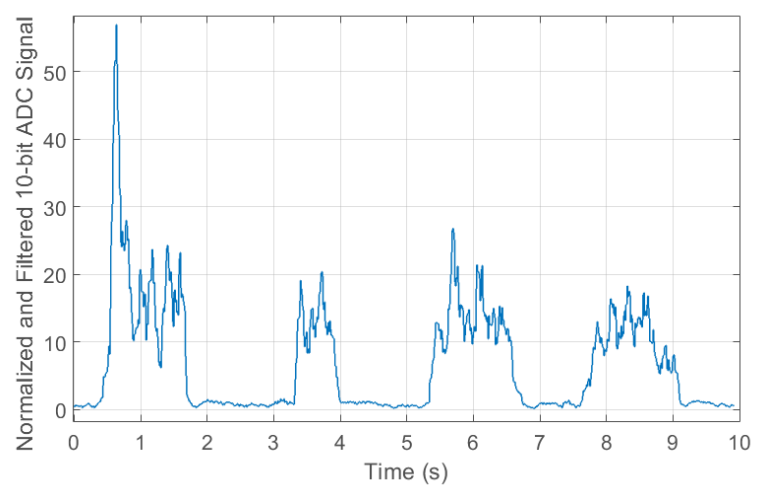

Fig. 8. Filtered sEMG Recording

The final step in the processing of the sEMG recording was to incorporate an Interval Test Block. This determines if the average value over a given amount of time is within set boundaries, producing a binary output. This boundary condition would be adjusted per the individual's recorded muscular activity. In instances where a child is first exposed to the system, a low sensitivity value might aid in initially presenting the function of the device, with the sensitivity later being reduced as the user familiarizes themselves with the system and its required muscle flexion/relaxation. The final binary output is shown in Figure 9, by comparing it to Figure 7 we can see that the periods of activity line up with the active 
phases in the raw sEMG recording.

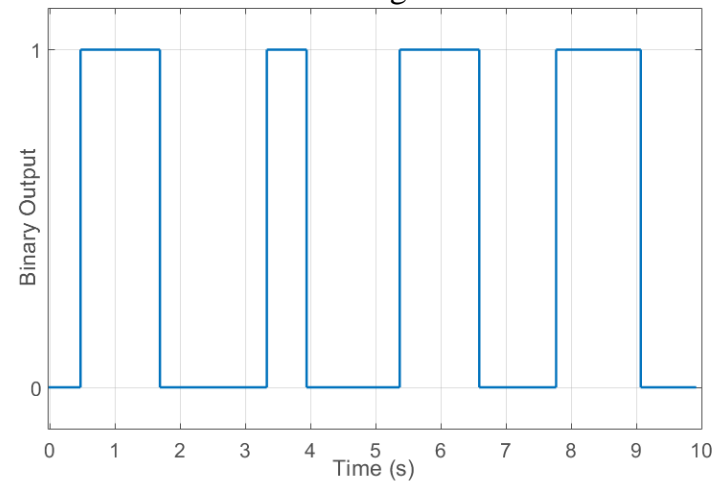

Fig. 9. Final Binary Output

When the sEMG system outputs a HIGH signal, the 'hand' begins to open and this will continue until the set maximum limit of the actuator is met. For the 'fingers,' the actuator traverses between $0 \mathrm{~mm}$ extension for fully closed and $19 \mathrm{~mm}$ for fully open. The 'thumb,' meanwhile, has limits of $5 \mathrm{~mm}$ to $19 \mathrm{~mm}$ for closed and open respectively. This open-close hand transition takes approximately $2.3 \mathrm{~s}$, with closed to open averaging $2.1 \mathrm{~s}$.

When the muscle is relaxed and the sEMG outputs a LOW signal, the hand will begin to close, ending once the limit is reached. If an obstruction is present during this process, such as one caused from grasping an object, the motor would ordinarily continue to be powered, draining the battery and reducing the life span of the actuator. For this reason, a method of obstruction detection has been employed.
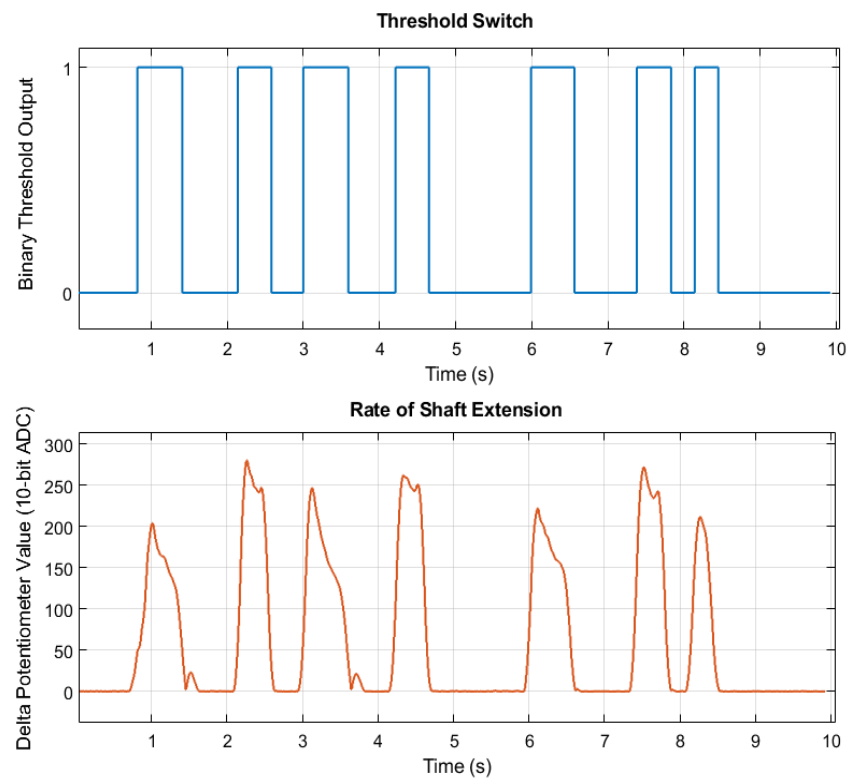

Fig. 10. Grasp Detection System: Threshold Switch (top), Rate of Shaft Extension (bottom)

The built-in potentiometer detects the position of the shaft: if the difference from a previous reading is considered, then the speed and direction of the shaft extension can be determined. The system utilizes this so that when an obstruction, i.e. a grasp, occurs and the speed of the shaft is slowed below a set value, the system shuts off the power to the motors to hold them in their current position. Figure 10 shows the speed of the shaft (bottom) and the binary output for this subsystem (top). The system can be adjusted so that even a slight obstruction causes the motors to stall. In its current setup, objects such as a soft toy will be detected as a grasp once the object has experienced a small amount of deformation.

\section{EXPERIMENTAL PROCEDURE AND RESULTS}

For the grasping experiments, the arm was connected to an Arduino Uno based circuit, running essentially the same script as shown in Section III. The only change is that the arm is controlled using buttons, rather than the sEMG, to simplify the experimental process. The tests also compared a variant of the hand that replaces the two segment 'thumb' with a three segment 'finger,' so that all the digits match.

\section{A. Object Grasps}

Twelve objects, shown in Figure 11, were selected to test the grasp effectiveness of the prosthetic. These objects range from items a toddler might interact with, such as toys, to geometric shapes designed to illustrate the range of grasps available on account of the soft grippers.

Each object was grasped 10 times and the failure or success noted, as well as the orientation of the object. For a grasp to be classified as successful, the object must remain stable in the 'hand' for a period of 10s whilst the arm is steadily shaken. This test was performed with both the two and three-segment 'thumb' variants, in order to facilitate comparison. The results of the test (Table I) show that across all the objects the three-segment 'thumb' performed slightly better on average, particularly with large objects or those with complex geometry, such as the set of keys.

TABLE I

RESULTS FROM OBJECT GRASP TESTS

\begin{tabular}{lccc}
\hline \multirow{2}{*}{ Test item } & $\begin{array}{c}\text { Object Mass } \\
\text { (g) }\end{array}$ & \multicolumn{2}{c}{ Grasp Success Rate } \\
\cline { 3 - 4 } & & Three Segment & Two Segment \\
\hline $\begin{array}{l}\text { Plastic water } \\
\text { bottle (empty) }\end{array}$ & 20.6 & $100 \%$ & $100 \%$ \\
\hline $\begin{array}{l}\text { Plastic water } \\
\text { bottle (250ml) }\end{array}$ & 270.6 & $100 \%$ & $100 \%$ \\
\hline Pen & 11.5 & $100 \%$ & $100 \%$ \\
\hline Wooden stick & 2.7 & $60 \%$ & $60 \%$ \\
\hline Sponge ball & 23.7 & $100 \%$ & $100 \%$ \\
\hline Set of Keys & 94.2 & $80 \%$ & $50 \%$ \\
\hline Soft Toy & 21.3 & $100 \%$ & $90 \%$ \\
\hline Hard Plastic Toy & 56 & $90 \%$ & $80 \%$ \\
\hline Cube & 31 & $100 \%$ & $100 \%$ \\
\hline Cone & 9 & $60 \%$ & $50 \%$ \\
\hline Pyramid & 13.2 & $60 \%$ & $60 \%$ \\
\hline Tri-Prism & 13.2 & $80 \%$ & $80 \%$ \\
\hline Cylinder & 20.4 & $100 \%$ & $100 \%$ \\
\hline Average & & $87 \%$ & $82 \%$ \\
\hline \hline
\end{tabular}




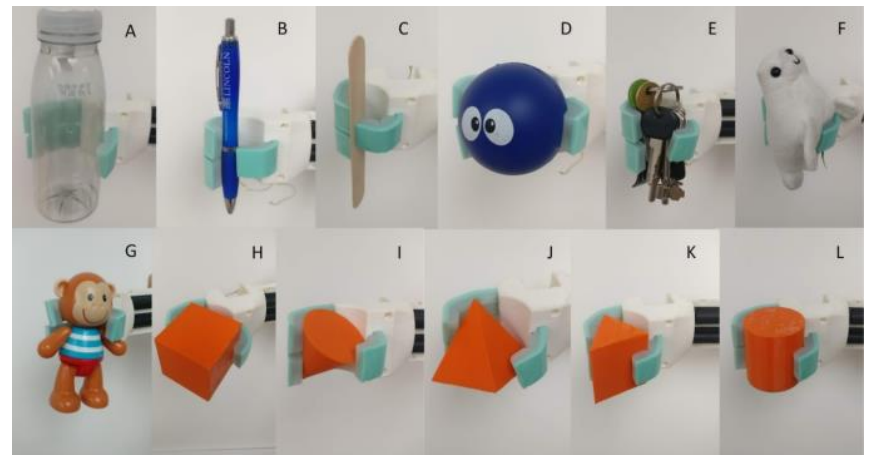

Fig. 11. Object grasp testing: Plastic Bottle (A), Pen (B), Wooden Stick (C), Sponge Ball (D), Set of Keys (E), Soft Toy (F), Hard Plastic Toy (G), Cube $(\mathrm{H})$, Cone (I), Pyramid (J), Triangular Prism (K), Cylinder (L)

\section{B. Grasping Force}

To determine the approximate grasping force of the hand, three methods have been employed. The first takes modified geometric objects from the previous subsection and attaches a series of weights to the grasped objects (Figure 12). The objects consist of $45 \mathrm{~mm}$ and $22.5 \mathrm{~mm}$ diameter cylinders, a triangular prism, and a $10 \mathrm{~mm}$ wide rectangular segment that is held in a pinch grasp. The point where the grippers experience lateral deformation, the point of slippage due to movement, and the point of absolute slippage when the arm is stationary (Figure 13) are documented. The tests compare the use of the two segment and three-segment 'thumb,' with the two-segment showing an increased grasping capability in three of the four shapes tested. This is likely down to the superior contact pad distribution seen when using the two-segment thumb.
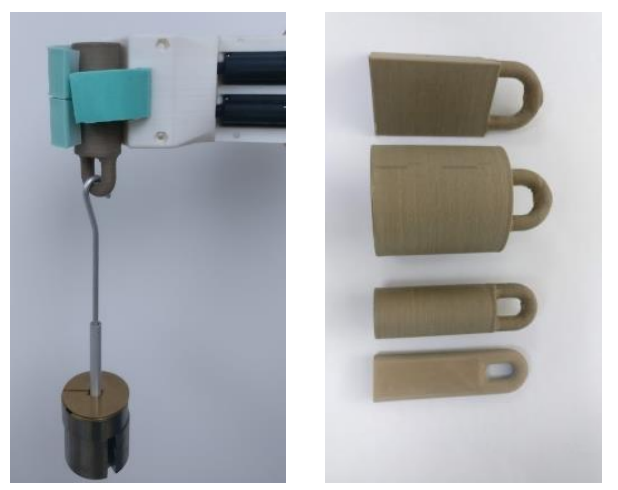

Fig. 12. 22.5mm Diameter Cylinder Weight Test (left), objects used in weight test (right)

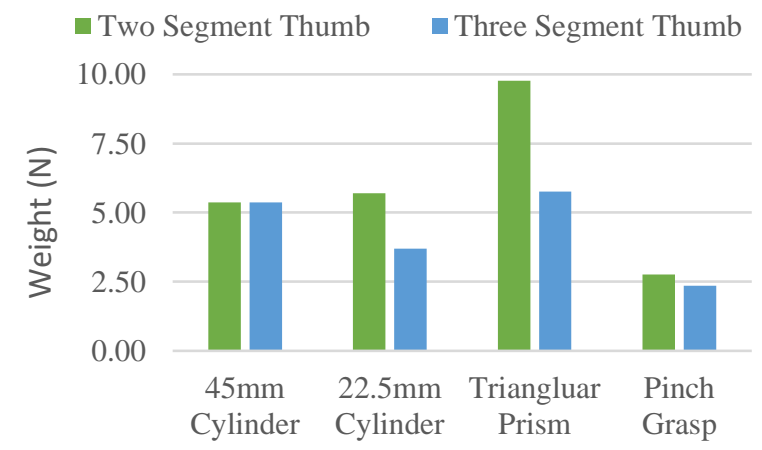

Fig. 13. Point of Absolute Slippage
The second test used a set of high accuracy scales to measure the approximate pinch force of the hand. The scales were rested on a raised platform, allowing the overhanging edge to be pinched by the grippers. This test was performed under three conditions: the motor powered, the motor unpowered, and the system with the grasp detection active. Each test was conducted 10 times, with the average force then being calculated (Table II.). The difference between the twosegment and three-segment 'thumb' was negligible. With the motor running, the highest pinch force of $8.5 \mathrm{~N}$ is noted. This is $35 \%(5.5 \mathrm{~N})$ higher than when the motor is unpowered, and $54.8 \%(3.9 \mathrm{~N})$ higher than when the grasp detection is active.

TABLE II

Mean AVERAGE Pinch Force

\begin{tabular}{lcc}
\hline \multicolumn{1}{c}{ Grasp Type } & Mass $(\mathrm{g})$ & Force $(\mathrm{N})$ \\
\hline Motor Powered & 869.8 & 8.5 \\
\hline Motor Unpowered & 565.1 & 5.5 \\
\hline $\begin{array}{l}\text { Grasp Detection } \\
\text { Active }\end{array}$ & 393 & 3.9 \\
\hline \hline
\end{tabular}

The final test method utilized the Takei Physical Fitness Test: Grip-A (Takei Scientific Instruments Co., Ltd). This test is designed to measure the grip force of biological hands. The size can be adjusted to accommodate child-size hands, allowing the prosthetic's grip force to be measured. The readings from the analogue dial, show around $0.5 \mathrm{~kg}(4.9 \mathrm{~N})$ when the maximum grip force is applied. With the grip detection system active, the reading is between $0.2 \mathrm{~kg}(1.96 \mathrm{~N})$ and $0.3 \mathrm{~kg}(2.94 \mathrm{~N})$. The test provides a guide to average grip strength by age: for a 4-year-old child the grip strength is given as $6.5 \mathrm{~kg}(63.8 \mathrm{~N})$ and $4.4 \mathrm{~kg}(43.2 \mathrm{~N})$, for males and females respectively. The main constraint on grasping force is the motor. Due to the tight size and weight restrictions in place, the grasping power will be limited. This continues to be an issue, even with larger adult devices.

\section{DISCUSSION AND CONCLUSIONS}

The work presented here demonstrates the viability of a prosthetic device at a scale suitable for toddlers. The design and manufacturing process utilized CAD and additive manufacturing as an alternative to the traditional method of producing prosthetic devices using techniques such as stump casting. The process of manufacturing prosthetic devices can be decentralized, utilizing a remote CAD designer in cases where the client is unable physically to visit a prosthetist, such as in low-income nations. The material cost of producing this device was around $£ 500$, including scrappages and prototypes. This represents a significant reduction in cost compared to the current production myoelectric devices, though it is worth noting that the overheads have not been considered here. The final cost on the device would vary slightly depending on the specific configuration based around the end user and the production scalability of standard parts, such as the grippers.

The outlined control system demonstrates that a simple system utilizing an armband sEMG device can produce consistent results. The linear actuator's built in potentiometer 
allows for a rudimentary grasp detection system to be integrated into the design of the prosthetic. The use of Simulink permits easy editing of the detection parameters as well as real-time monitoring to assist in determining them. The system has only been tested on an able-bodied adult, and further investigation is required to determine the effectiveness of the system when used with a child presenting an upper limb reduction, due to the expectedly weaker sEMG signal.

From the experimental data a mean average grasp effectiveness of $87 \%$ and $82 \%$, for the three and two-segment 'thumb' respectively, has been demonstrated. The close performance of the variants leads to the decision to use the two-segment 'thumb,' as it is a more cosmetically pleasing design. The malleable nature of the grippers has been shown to be effective in these tests: this is twofold, primarily by providing a supple contact surface that mimics the feel of human skin, whilst secondarily allowing the grippers to deform laterally, providing a more encompassing grasp as presented in Figure 11.

The weighted tests too show the effectiveness of the prosthetic, with masses under $400 \mathrm{~g}$ proving to be stable in all but the pinch grasp, where slippage under movement occurs at $220 \mathrm{~g}$. This is due to the lateral deformation in the grips caused by the moment acting on the pinch point. This highlights a potentially limiting factor with the design of the grippers, should this style of gripper be utilized. The pinch grip tests give a maximum force between $8.5 \mathrm{~N}$ and $3.9 \mathrm{~N}$, depending on the configuration: this - when compared to published literature on the pinch force of adult prosthetic hands - demonstrates that the presented device performs competitively, with pinch strength ranging between $1.71 \mathrm{~N}$ and $16.11 \mathrm{~N}$ [20]. The Takei test gives a maximum force of $4.9 \mathrm{~N}$ : comparing this to the manufacturer's given data of $63.8 \mathrm{~N}$ for a 4 -year-old boy, represents a $92.3 \%$ reduction in grip force. The cause for this is primarily the actuators, which individually produce a maximum rated force of $30 \mathrm{~N}$. As the device is designed for toddlers, there are strict size limitations on all components, including the actuators. Even within larger adult devices, grip strength proves to be an issue. However, the use of an improved end device such as the presented soft grippers, aims to increase the grasp effectiveness by negating the need to rely on shear grip force to securely grasp objects.

The aim of this project was to showcase the feasibility of a 3D-printed upper limb prosthetic device for toddlers that utilizes soft grippers. In this endeavor great promise has been shown, with the final device performing well in the devised tests. The reduction in lead time and financial cost demonstrated by the presented design opens up the possibility of such a device becoming available from healthcare providers in high-income nations. Correspondingly, in low-income nations, there is the opportunity for adoption due to the decentralized and low-cost nature of 3D-printing techniques.

This initial design is used as a proof of concept. Due to restrictions on the project, the device has not been verified on the target audience of young children. The next stage would be to focus on end-user engagement, which would include qualitative data around functionality, acceptability, and cosmetic appearance. Subsequent design improvements would then be based on the results of such a study.

\section{REFERENCES}

[1] E. Vasluian, C. K. Van Der Sluis, A. J. Van Essen, J. E. H. Bergman, P. U. Dijkstra, H. A. Reinders-Messelink, and H. E. K. De Walle, "Birth prevalence for congenital limb defects in the northern Netherlands: A 30-year population-based study," BMC Musculoskelet. Disord., vol. 14, 2013.

[2] T. R. Dillingham, L. E. Pezzin, and E. J. MacKenzie, "Limb amputation and limb deficiency: Epidemiology and recent trends in the United States," South. Med. J., vol. 95, no. 8, pp. 875-883, 2002.

[3] M. Egermann, P. Kasten, and M. Thomsen, "Myoelectric hand prostheses in very young children," Int. Orthop., vol. 33, no. 4, pp. 1101-1105, 2009.

[4] H. Mano, S. Fujiwara, and N. Haga, "Adaptive behaviour and motor skills in children with upper limb deficiency," Prosthet. Orthot. Int., vol. 42, no. 2, pp. 236-240, 2018.

[5] M. Allami, B. Mousavi, M. Masoumi, E. Modirian, H. Shojaei, F. Mirsalimi, M. Hosseini, and P. Pirouzi, "A comprehensive musculoskeletal and peripheral nervous system assessment of warrelated bilateral upper extremity amputees," Mil. Med. Res., vol. 3, no. 1, pp. 1-8, 2016.

[6] S. Postema, Upper limb absence: Effects on body functions and structures, musculoskeletal complaints, and functional capacity. 2017.

[7] M. Meurs, C. G. B. Maathuis, C. Lucas, M. Hadders-Algra, and C. K. van der Sluis, "Prescription of the first prosthesis and later use in children with congenital unilateral upper limb deficiency: A systematic review," Prosthet. Orthot. Int., vol. 30, no. 2, pp. 165-173, 2006.

[8] T. Scotland and H. Galway, "A long-term review of children with congenital and acquired upper limb deficiency," J. Bone Joint Surg. Br., vol. 65-B, no. 3, pp. 346-349, 1983.

[9] K. Postema, V. Van Der Donk, J. Van Limbeek, R. A. J. Rijken, and M. J. Poelma, "Prosthesis rejection in children with a unilateral congenital arm defect," Clin. Rehabil., vol. 13, no. 3, pp. 243-249, 1999.

[10] G. McGimpsey and T. Bradford, "Limb Prosthetics Services and Devices: Critical Unmet Need: Market Analysis,” Bioeng. Inst. Cent. Neuroprosthetics, pp. 1-35, 2017.

[11] Nicolas E. Walsh and Wendy S. Walsh, "Developing, war-torn countries-with damaged infrastruc-tures at government and community levels-lack systems for rehabilitation," vol. 81, no. 3, pp. 665-670, 2003.

[12] M. Yoshikawa, R. Sato, T. Higashihara, T. Ogasawara, and N. Kawashima, "Rehand: Realistic electric prosthetic hand created with a 3D printer," Proc. Annu. Int. Conf. IEEE Eng. Med. Biol. Soc. EMBS, vol. 2015-Novem, pp. 2470-2473, 2015.

[13] M. King, B. Phillips, M. Shively, V. Raman, A. Fleishman, S. Ritter, and K. Mehta, "Optimization of prosthetic hand manufacturing," Proc. 5th IEEE Glob. Humanit. Technol. Conf. GHTC 2015, vol. 6, pp. 59-65, 2015.

[14] J. ten Kate, G. Smit, and P. Breedveld, "3D-printed upper limb prostheses: a review," Disabil. Rehabil. Assist. Technol., vol. 12, no. 3, pp. 300-314, 2017.

[15] A. Kargov, C. Pylatiuk, J. Martin, S. Schulz, and L. Döderlein, “A comparison of the grip force distribution in natural hands and in prosthetic hands," Disabil. Rehabil., vol. 26, no. 12, pp. 705-711, 2004.

[16] J. Lenarčič, T. Bajd, and M. M. Stanišić, "Robot Grasp," in Robot Mechanisms, 2013, pp. 291-311.

[17] S. Plagenhoef, F. G. Evans, and T. Abdelnour, "Anatomical Data for Analyzing Human Motion," Res. Q. Exerc. Sport, vol. 54, no. 2, pp. 169-178, Jun. 1983.

[18] O. Neyzi, R. Bundak, G. Gökçay, H. Günöz, A. Furman, F. Darendeliler, and F. Baş, "Reference values for weight, height, head circumference, and body mass index in Turkish children," JCRPE J. Clin. Res. Pediatr. Endocrinol., vol. 7, no. 4, pp. 280-293, 2015.

[19] A. Muzumdar, Powered Upper Limb Prostheses: Control, Implementation and Clinical Application. Berlin, 2004.

[20] P. Slade, A. Akhtar, M. Nguyen, and T. Bretl, "Tact: Design and performance of an open-source, affordable, myoelectric prosthetic hand," Proc. - IEEE Int. Conf. Robot. Autom., vol. 2015-June, no. June, pp. 6451-6456, 2015. 